\title{
Genome wide identification and predicted functional analyses of NAC transcription factors in Asian pears
}

\author{
Mudassar Ahmad ${ }^{1,2,3}$, Xinhui Yan ${ }^{1,2,3}$, Jianzhao Li ${ }^{1,2,3}$, Qinsong Yang ${ }^{1,2,3}$, Wajeeha Jamil ${ }^{1,2,3}$, Yuanwen Teng ${ }^{1,2,3^{*}}$ \\ and Songling $\mathrm{Bai}^{1,2,3^{*}}$ (D)
}

\begin{abstract}
Background: NAC proteins contribute to diverse plant developmental processes as well as tolerances to biotic and abiotic stresses. The pear genome had been decoded and provided the basis for the genome-wide analysis to find the evolution, duplication, gene structures and predicted functions of PpNAC transcription factors.

Results: A total of 185 PpNAC genes were found in pear, of which 148 were mapped on chromosomes while 37 were on unanchored scaffolds. Phylogeny split the NAC genes into 6 clades (Group1- Group6) with their sub clades ( subgroup A to subgroup $H$ ) and each group displayed common motifs with no/minor change. The numbers of exons in each group varied from 1 to 12 with an average of 3 while 44 pairs from all groups showed their duplication events. QPCR and RNA-Seq data analyses in different pear cultivars/species revealed some predicted functions of PpNAC genes i.e. PpNACs 37, 61, 70 (2A), 53, 151(2D), 10, 92, 130 and 154 (3D) were potentially involved in bud endodormancy, PpNACs 61, 70 (2A), 172, 176 and 23 (4E) were associated with fruit pigmentations in blue light, PpNACs 127 (1E), $46(1 \mathrm{G})$ and $56(5 \mathrm{~A})$ might be related to early, middle and late fruit developments respectively. Besides, all genes from subgroups $2 \mathrm{D}$ and $3 \mathrm{D}$ were found to be related with abiotic stress (cold, salt and drought) tolerances by targeting the stress responsive genes in pear.

Conclusions: The present genome-wide analysis provided valuable information for understanding the classification, motif and gene structure, evolution and predicted functions of NAC gene family in pear as well as in higher plants. NAC TFs play diverse and multifunctional roles in biotic and abiotic stresses, growth and development and fruit ripening and pigmentation through multiple pathways in pear.
\end{abstract}

Keywords: Pear, Genome-wide analysis, NAC transcription factors, PpNAC predicted functions, Bud endodormancy, Fruit color, Fruit development

\section{Background}

Transcription factors are master regulators which mediate transcriptional regulations by binding a specific nucleotide sequence in response to developmental and environmental changes in plants. As a crucial type of TFs, NAC, a term derived from 3 genes i.e. NAM (No apical meristem), ATAF $1 / 2$ (Arabidopsis transcription activator factor $1 / 2$ ) and CUC2 (cup shaped cotyledon) [1], is not only one of the largest transcription factors, but also has an important

\footnotetext{
*Correspondence: ywteng@zju.edu.cn; songlingbai@zju.edu.cn 'Department of Horticulture, Zhejiang University, Hangzhou 310058, Zhejiang, China

Full list of author information is available at the end of the article
}

position in plant developments and stress regulations [2]. Protein sequences of this family indicate that the $\mathrm{N}$-terminal region (NAC domain) of these proteins is highly conserved and comprises about 160 amino acid residues and involve in DNA binding while the $\mathrm{C}$-terminal region is highly diversified in length and sequence and consider as the transcriptional activation domain [3]. The $\mathrm{N}$-terminal region (NAC domain) is further divided into 5 subdomains (A-E) of conserved blocks embedded in heterogeneous blocks or gaps. "A" subdomain promotes functional dimerization, "B" and " $E$ " has distinctive functions of protein while "C" and " $\mathrm{D}$ " are positively charged and allow the TF to bind to the DNA [4-7].

(c) The Author(s). 2018 Open Access This article is distributed under the terms of the Creative Commons Attribution 4.0 International License (http://creativecommons.org/licenses/by/4.0/), which permits unrestricted use, distribution, and 
More than 100 members of NAC TFs are extensively distributed in the plant genome while a few of them are found to have diverse biological processes, including formation and maintenance of shoot apical meristem [8], embryo, seed and lateral root developments [9-11], hormone signaling [12], cell division [13], regulation of secondary cell wall synthesis [14] and fruit ripening, growth and coloration $[15,16]$. In particular, NAC TFs have got much attention as regulators in plant tolerance pathways during both biotic and abiotic stresses. For instance, AtAF1 and AtAF2 related to fungal diseases in Arabidopsis [17], OsNAC6 enhanced high salinity, dehydration and disease tolerances in transgenic rice plants [18], StNAC gene was related to pathogen attack and wounding in potato [19], drought and cold induced $B n N A C$ genes in Brassica [20], SNAC1/2 helped in drought resistance and salt tolerance in rice $[21,22]$ and overexpression of AtNAC019, AtNACO72, and AtNAC055 increased drought and stress tolerances [6] while AtNAC2 enhanced salt tolerance through ethylene signaling pathway in Arabidopsis [23]. These biotic and abiotic stresses are the major factors that cause limiting growth and productivity [24] and NAC TFs are up and down regulated during these limitations in both herbaceous as well as woody plants.

Pear from Rosaceae family is one of the most important temperate deciduous perennial woody plants. The NAC genes may play fundamental roles on the development and biotic/abiotic stresses in pear and other rosaceous plants as well. As in pear, PbeNAC1 was involved in cold and drought tolerances via modulating the expression of stress-responsive genes (NtRD29A, NtRD17 etc), biosynthesis of proline (NtP5CS) and ROS scavenging enzymes (NtCAT, NtSOD, and NtAPX) in transgenic tobacco through interacting with dormancy related PbeDREB1 and PbeDREB2A genes [25]. In apple, MdNACO29 negatively regulated cold tolerance via CBF-dependent pathway [26] while MdNAC047 enhanced the salt stress tolerance by modulating the ethylene response [27]. NAC TF was also be found to be highly overexpressed in blood-fleshed peaches as compared to non-red-fleshed peaches and Ppe$N A C 1$ helped in anthocyanin accumulation in tobacco by interacting with PpeMYB10 [16].

To date, genome wide analyses of NAC TFs have been investigated in various plant species such as Arabidopsis [3], rice [28], grapes [29], tobacco [30], soybean [31], apple [32], cabbage and wheat [33]. We performed the genome wide and predicted functional analyses of PpNAC TFs based on the draft genome sequence of pear [34]. Given the importance of this family, the chromosome location, gene structures and protein motifs of the putative PpNAC genes predicted by genome wide surveys were carefully analyzed. The putative pear NAC genes were further subjected to phylogenetic analyses with Arabidopsis and apple. These comparisons enabled the identification of genes for their further characterizations. Moreover, we paid additional attentions to find the predicted $P p N A C$ genes related to stress tolerance, bud endodormancy, fruit pigmentation and fruit development.

\section{Results}

\section{Identification of NAC gene family in pear}

The first strategy of Hidden Markov Model search (HMM search) with the HMM profile (PF01849) of the NAM domain against the pear predicted protein database and the other strategy of plant transcription factor database resulted in $230 P p N A C$ proteins. The sequences which were redundant, overlapped, incomplete and repeated were rejected and further manually analyzed using Interproscan and SMART blast to reconfirm the presence of the NAM domains in each sequence. Finally, 185 sequences containing typical NAM domain with full ORFs were identified and considered as the pear NAC transcription factors. The detailed information and protein sequences of selected pear NAC TFs is listed in Additional file 1.

\section{Phylogenetic analysis of PpNAC TFs}

To explore the phylogenetic relationships among 185 $P p N A C$ genes and to clarify the evolutionary relations within this family, a combined phylogenetic tree of pear was constructed with apple and Arabidopsis NAC proteins. Due to high diversity in sequence lengths, a maximum likelihood phylogenetic tree was constructed which split the NAC proteins into 6 distinct clades (Group1- Group6) with their sub clades ( subgroup A to subgroup H) (Fig. 1). Group 1 was the largest one with 40 PpNAC genes and 8 subgroups $(1 \mathrm{~A}-1 \mathrm{H})$ followed by groups 2 and 6 with 34 genes of each and 4 (2A-2D) and 3 (6A-6C) subgroups respectively (Table 1). Further, members of $P p N A C$ TFs in groups $1,2,3$ and 5 were clustered with the members of apple and Arabidopsis although some species-specific subgroups were also been identified (subgroup 3A). In contrary, genes of pear and apple in groups 4 and 6 were separately clustered with those of Arabidopsis and demonstrated some independent evolutionary history between Arabidopsis and Rosacea plants. Detailed phylogenetic tree of apple, pear and Arabidopsis is in Additional file 2.

Previous studies on NAC TFs demonstrated that genes clustered in one subgroup might have tendency to do similar functions. So, we searched some predicted functions of PpNAC groups based on the published papers. Our findings revealed that group 1 might belong to cell growth and development while group 2, 3 and 5 performed some signaling roles during biotic and 


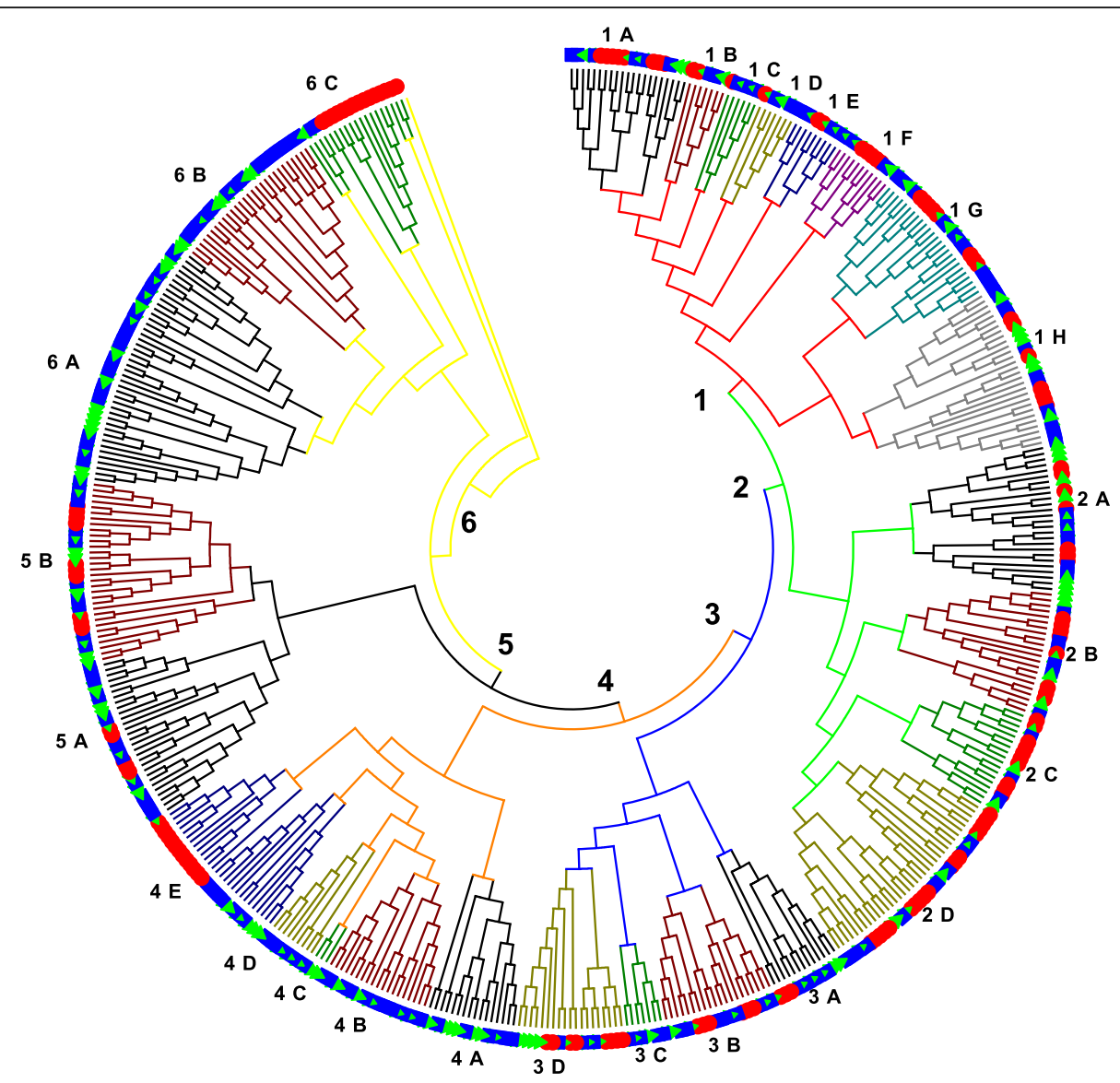

Fig. 1 Phylogenetic analysis of the NAC transcription factors of Arabidopsis, apple and pear represented by red circles, blue squares and green triangles respectively. The full-length sequences of the NAC proteins were aligned using MUSCLE, and the phylogenetic tree was made using the maximum-likelihood tree method in the MEGA7.0 software. The tree was divided into 6 main groups 1, 2, 3, 4, 5 and 6 represented by red, green, blue, orange, black and yellow colored clusters within the tree while their subgroups designated from $1 \mathrm{~A}-1 \mathrm{H}, 2 \mathrm{~A}-2 \mathrm{D}, 3 \mathrm{~A}-3 \mathrm{D}, 4 \mathrm{~A}-4 \mathrm{E}, 5 \mathrm{~A}-5 \mathrm{~B}$ and $6 \mathrm{~A}-6 \mathrm{C}$ respectively outside the tree

abiotic stresses. Although there were some specificity in predicted functions at subgroup and gene levels in PpNAC TFs as shown in Table 1. But, functional evaluations of NACs from groups 4 and 6 were hard to be predicted due to independent evolution (Fig. 1 \& Table 1).

\section{Genome distribution and duplication analysis of PpNAC gene family}

The chromosome locations of all PpNAC genes were identified using BLAST software 2.25 to align these $P p N A C$ sequences against the genome sequences of pear. The physical map positions demonstrated that in total, 148 (81\%) PpNAC genes were localized on 16 chromosomes in ascending order from short arm telomere to long arm telomere and interestingly chromosome 2 was unoccupied by $P p N A C$ genes (Fig. 2a). The remaining 37 genes could not be conclusively charted on chromosomes due to presence on unanchored scaffolds, so they were mapped on Cun1 (11\%) and Cun2 (8\%) in ascending order. The given names of PpNAC TFs were according to these ascending order arrangements in chromosomes as well as Cuns. The position and length of each gene are shown in Additional file 1. Maximum $P p N A C$ gene frequencies were observed on chromosome 11 (11\%) followed by chromosome 10 (9\%) while minimum frequencies were shown by chromosome 16 and 7 (1\% and 1.6\%) (Fig. 2b; Additional file 3). The maximum percentage of groups 1, 2, 3, 4, 5 and 6 were located on chromosome 10 (17.5\%), chromosome 10 (17.6\%), chromosome 11 (17\%), chromosome 4 (13\%), chromosome 15 (16\%) and chromosome 11 (38\%) respectively while group 4 were also highly mapped on Cun1 (33\%) and Cun2 (23\%) (Additional file 3).

Due to the presence of a whole-genome duplication event in the evolution of pear, gene duplication events within pear NAC family had also been detected as similar in several plants. To find out the duplication events in PpNAC TFs, a collinerity analysis had been performed by using MCScanX software. In $185 \mathrm{PpNAC}$ TFs, we found 44 pairs of duplicated genes (Fig. 3a, b). Maximum duplication events were seen in group 1 
Table 1 Group wise distributions and predicted functions of PpNAC transcription factors

\begin{tabular}{|c|c|c|c|c|c|c|c|}
\hline GROUP\# & Subgroups & No. of genes & Exons \# & Domain\# & Best Smart hit & Submitter description & Reference \\
\hline \multirow[t]{9}{*}{ GROUP1 } & $1 \mathrm{~A}$ & 7 & 3 & $1,4,2,5,3,6,7$ & NAC100 & Cell expansions & {$[40]$} \\
\hline & $1 \mathrm{~B}$ & 3 & & $1,2,5,3,6,7$ & CUC2 & Regeneration in root explants & {$[60]$} \\
\hline & $1 C$ & 2 & & $1,4,2,5,3,6,7$ & CUC3 & \multirow[t]{4}{*}{ Anthocyanin biosynthesis in peach } & \multirow[t]{4}{*}[16]{} \\
\hline & $1 \mathrm{D}$ & 3 & & $1,4,2,5,7$ & CUC1 & & \\
\hline & $1 \mathrm{E}$ & 3 & & $1,4,2,5,3,6,7$ & CUC2 LIKE & & \\
\hline & $1 \mathrm{~F}$ & 4 & & $1,4,2,5,3,6,7$ & NAC21/22 & & \\
\hline & $1 \mathrm{G}$ & 7 & & $1,4,2,5,3,6,7$ & BEARSKIN2 LIKE & Root cap maturation & {$[49]$} \\
\hline & $1 \mathrm{H}$ & 11 & & $1,4,2,5,3$ & VND & $\begin{array}{l}\text { Vascular and secondary cell wall } \\
\text { formation }\end{array}$ & {$[50]$} \\
\hline & Subtotal & 40 & & & & & \\
\hline \multirow[t]{5}{*}{ GROUP2 } & $2 \mathrm{~A}$ & 13 & 3 & $1,4,2,5,3,6,7$ & JUNGBRUNNEN 1-like isoform & \multirow[t]{2}{*}{ GA and BR signaling } & \multirow[t]{2}{*}[41]{} \\
\hline & $2 B$ & 8 & 6 & $1,4,2,5,3,6,7$ & NAC28 & & \\
\hline & $2 C$ & 5 & 5 & $1,4,2,5,3,6,7$ & NTM1, TCV & Salt stress response & {$[42]$} \\
\hline & $2 \mathrm{D}$ & 8 & 5 & $1,4,2,5,3,6,7$ & NTL 8 & Seed germination during salt stress & {$[42]$} \\
\hline & Subtotal & 34 & & & & & \\
\hline \multirow[t]{5}{*}{ GROUP3 } & $3 \mathrm{~A}$ & 6 & 3 & $1,4,2,5,3,6,7$ & NAC83 & & \\
\hline & 3B & 6 & 3 & $1,4,2,5,3,6,7$ & NAC2 & Stamen development in arabidopsis & {$[61]$} \\
\hline & $3 C$ & 3 & 2 & $1,4,2,5,3,6,7$ & NARS1 & Embryogenesis & {$[62]$} \\
\hline & $3 \mathrm{D}$ & 8 & 3 & $1,4,2,5,3,6$ & AtAF1 & Salt stress and ABA signaling in rice & {$[63]$} \\
\hline & Subtotal & 23 & & & & & \\
\hline \multirow[t]{6}{*}{ GROUP4 } & $4 \mathrm{~A}$ & 10 & 3 & $1,5,3,7$ & NAC67 & \multirow{6}{*}{\multicolumn{2}{|c|}{$\begin{array}{l}\text { Group4 genes in PpNAC and MdNAC TFs showed } \\
\text { independent evolution with AtNAC TFs. } \\
\text { Anthocyanin accumulation in pear (Present Study) }\end{array}$}} \\
\hline & $4 \mathrm{~B}$ & 7 & 6 & $1,4,2,5,3,6$ & NTM1 like & & \\
\hline & $4 C$ & 3 & 6 & $1,4,2,5,3,7$ & NAC isoform $X 1$ & & \\
\hline & $4 \mathrm{D}$ & 3 & 6 & $1,4,2,5,3,6,7$ & NAC83 like & & \\
\hline & $4 \mathrm{E}$ & 7 & 4 & $1,4,5,3,6,7$ & NAC14 & & \\
\hline & Subtotal & 30 & & & & & \\
\hline \multirow[t]{3}{*}{ GROUP5 } & $5 A$ & 10 & 3 & $1,4,5,3,6,7$ & AtNAP & Fruit and Leaf senescence & {$[64]$} \\
\hline & $5 B$ & 14 & $3 \& 8$ & $1,2,6,7$ & AtVNI2 & Abscisic acid signaling & {$[65]$} \\
\hline & Subtotal & 24 & & & & & \\
\hline \multirow[t]{4}{*}{ GROUP6 } & $6 \mathrm{~A}$ & 22 & 1, NO INTRON & $1,8,9,10,6,7$ & NAC41/CUC1 like & \multirow{4}{*}{\multicolumn{2}{|c|}{$\begin{array}{l}\text { Group6 genes in PpNAC and MdNAC TFs showed } \\
\text { independent evolution with AtNAC TFs }\end{array}$}} \\
\hline & $6 B$ & 10 & 2 & $1,8,2,6,7$ & NAC30 & & \\
\hline & $6 C$ & 2 & 3 & 1,7 & NAC30 like & & \\
\hline & Subtotal & 34 & & & & & \\
\hline TOTAL & & 185 & & & & & \\
\hline
\end{tabular}

followed by group 2 while groups 4 and 6 showed very less duplication events (Fig. 3b).

\section{Gene structure and protein motifs of PpNAC TFs}

Among the NAC family, the closest members in the same subgroups shared a similar exon/intron structures in terms of intron numbers and exon lengths (Fig. 4a, b). For instance, group A with all its subgroups had 3 exonic and 2 intronic regions and subgroup 6 had no or one intronic region and seemed to be the smallest in structure than all others. Group 5 possessed the biggest structure and most numbers of exon and intron in their members (Fig. 4b). In contrast, members of subgroups $2 \mathrm{~B}, 2 \mathrm{C}, 4 \mathrm{C}, 4 \mathrm{E}$, and $5 \mathrm{~B}$ showed a large variability in either the distribution or number of introns (Table 1 ). If specifically highlighted, PpNAC 148 (5B) had the biggest gene structure with 12 intronic parts followed by PpNAC 23 (4E) and PpNAC 47 (2C) while PpNAC 76 (4C) had the biggest intron structure than others (Fig. 4b). In short, the coding regions in PpNAC TFs varied from 1 to 12 with an average of 3 as found in NAC population of other species. These gene structures also 


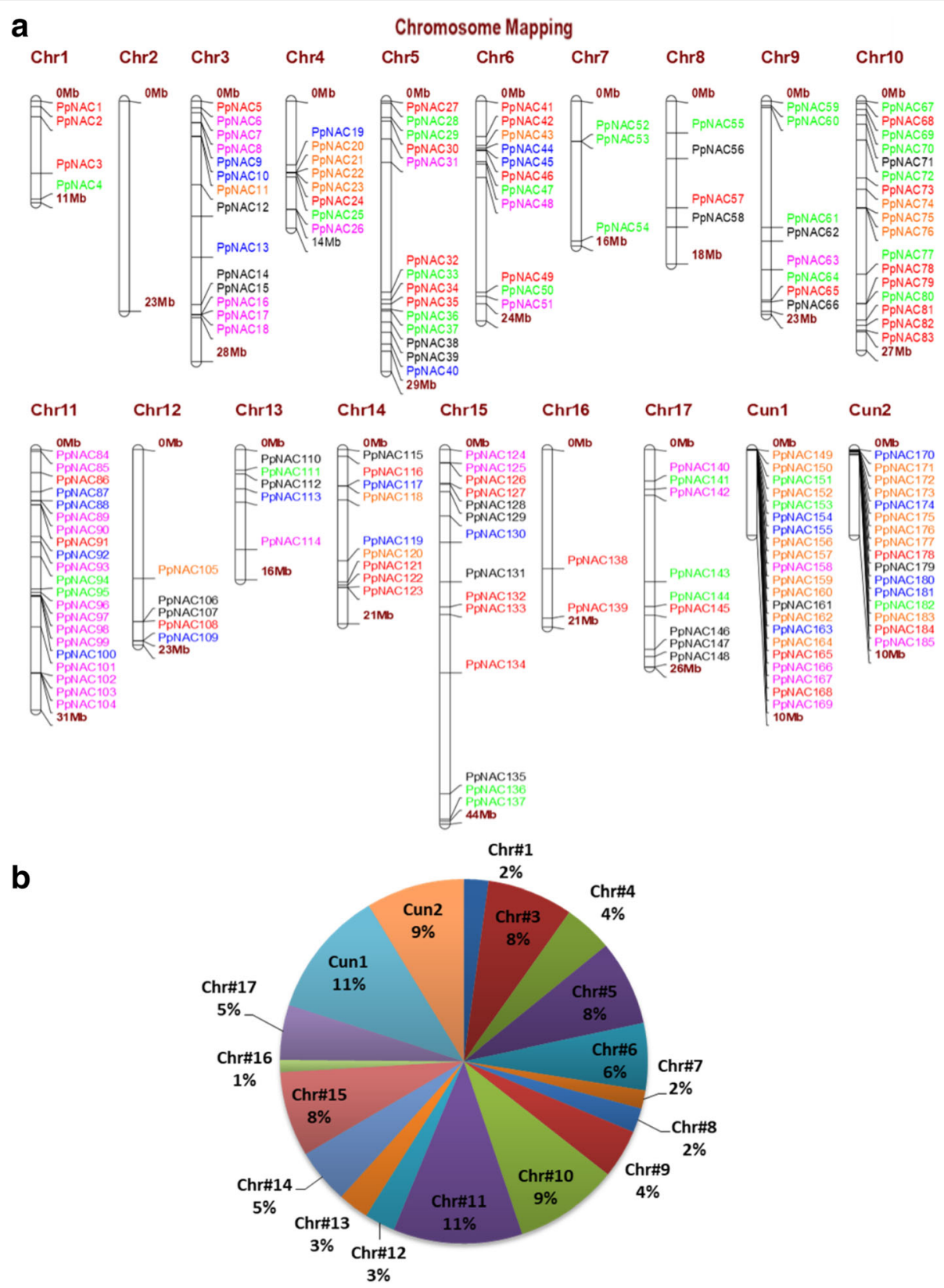

Fig. 2 Chromosomal mapping and frequency distribution of the PPNAC transcription factors. a, Physical locations of the putative PpNAC genes on each chromosome. Red, green, blue, orange, black and purple indicate groups 1, 2, 3, 4, 5 and 6 respectively. The chromosomal length is shown at the end while the chromosome number (Chr01-Chr17) is shining at the top. PpNAC genes located on scaffold are mapped on Cun1 and Cun2 with $10 \mathrm{Mb}$ lengths. $\mathbf{b}$, Number of PpNAC genes according to their frequency distributions on each chromosome. Each color shows different chromosome numbers

provided the reliable substantiation to support and authenticate our phylogenetic groupings and subgroupings in PpNAC genes.

To further explore the diversification and potential conserved motifs in PpNAC genes, putative motifs were predicted by the Multiple Em for Motif Elicitation (MEME) tool and 10 divergent motifs were recognized in PpNAC TFs and named as motifs 1-10 (Fig. 4c). Motif 1 is recognized as NAM while others (Motifs 2-10) were remained unrecognized. As expected, we found that most of the groups based on the phylogeny displayed common motifs with same alignment and position while there were no/minor differences at subgroup levels. So, NAC proteins with similar gene structures and motifs were present in the same subgroups and might have the similar functions as shown in Table 1. 


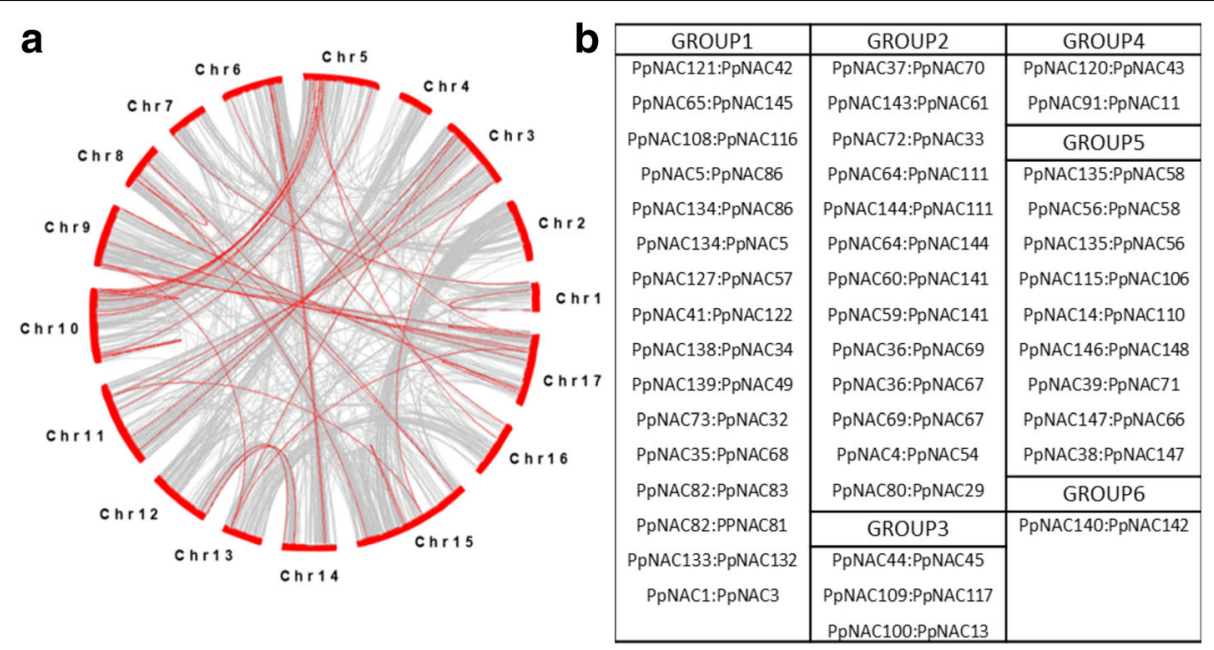

Fig. 3 Collinearity analysis of PpNAC genes. a, Red lines indicate the PpNAC genes duplication while the gray lines indicate the whole genome duplication events. Red bars indicate each chromosome number. b. Duplicated gene pairs of PpNAC TFs according to their assigned groups

\section{Expression analysis of PpNAC genes based on RNA-Seq datasets}

To find out the possible roles of PpNAC genes, their expression profiles were analyzed based on the three published RNA-Seq datasets: (i) pear bud dormancy in Pyrus pyrifolia 'Suli' [35]; (ii) fruit color development in P. pyrifolia 'Meirensu' [36] and (iii) different fruit development stages in P. pashia, P. calleryana, and P. pyrifolia [37]. The expression patterns of PpNAC genes were classified into three types based on their expression profiles i.e. constitutive expressions, period specific expressions and no expressions.

(i) By comparing the RNA-seq data of flower buds during dormancy period, we identified 55 constitutive expressed genes from groups 1-5 while group 6 showed no expressions during the entire endodormancy cycle in 'Suli' pear (Additional file 4). We selected 19 differentially expressed genes mostly from subgroups $2 \mathrm{~A}, 2 \mathrm{C}, 2 \mathrm{D}$ and $3 \mathrm{D}$ with period specific expressions during December and January when chilling accumulation and endodormancy release step proceed in 'Suli' pear as found in our previous study [35]. So to reconfirm their relations with bud endodormancy, the expression analysis was performed of all genes from subgroups $2 \mathrm{D}$ and $3 \mathrm{D}$ and randomly selected from subgroup 2A during flower bud endodormancy from October 15 to Feb 5 with 15 days interval in P. pyrifolia 'Cuiguan' (Fig. 5B). Expression results revealed that $P p N A C s 37,61,70$ (2A), 53, 151(2D), 10, 92, 130 and 154 (3D) showed peaks in December and January and suggested that these $P p N A C$ genes might be related to bud endodormancy (Fig. 5B-a, b, c) in 'Cuiguan' pear. Besides, stress related dehydrin genes which protect the cells from dehydration and desiccation damages by the environment during the dormancy process also showed high expressions in P. pyrifolia 'Cuiguan' during bud endodormancy period (Fig. 5B-d).

(ii) The transcriptomic data during color development in fruit of $P$. pyrifolia 'Meirensu' revealed 42 constitutive expressed $P p N A C$ genes from groups 1-5 with some variations within groups and subgroups while there were no expressions in group 6 (Additional file 5). About 15 PpNAC genes showed period specific expressions after 1 day and 6 days (T7-T9 and T10-T12) under sunlight exposure and these genes were mostly from subgroups $1 \mathrm{C}, 2 \mathrm{~A}$ and $4 \mathrm{E}$ (Fig. 6a). So, we randomly selected 2 and 3 genes for expression analysis from subgroups 2A (PpNAC61, PpNAC70) and 4E (PpNAC172, PpNAC176, PpNAC23) respectively for expression analysis. The qPCR results exposed that above NAC TFs, under blue light and dark treatments with 0 h, 12 h, 1 day, 3 days, 6 days and 10 days interval revealed some relations with anthocyanin accumulations in pear fruit as the kinetic of coloration and anthocyanin accumulation were already been recorded in our previous study [38]. The expressions of PpNACs 61, 70, 172 and 176 were the highest after 1 day while sudden down after 3 and 6 days of blue light exposure as compared to dark conditions. In contrast, $P p N A C$ 23 showed low expressions at initial exposure days and high expressions with increasing exposure days of blue light than dark conditions (Fig. 6b). In conclusion, our results from RNA-seq and qPCR revealed that some genes of subgroups $2 \mathrm{~A}$ and $4 \mathrm{E}$ 


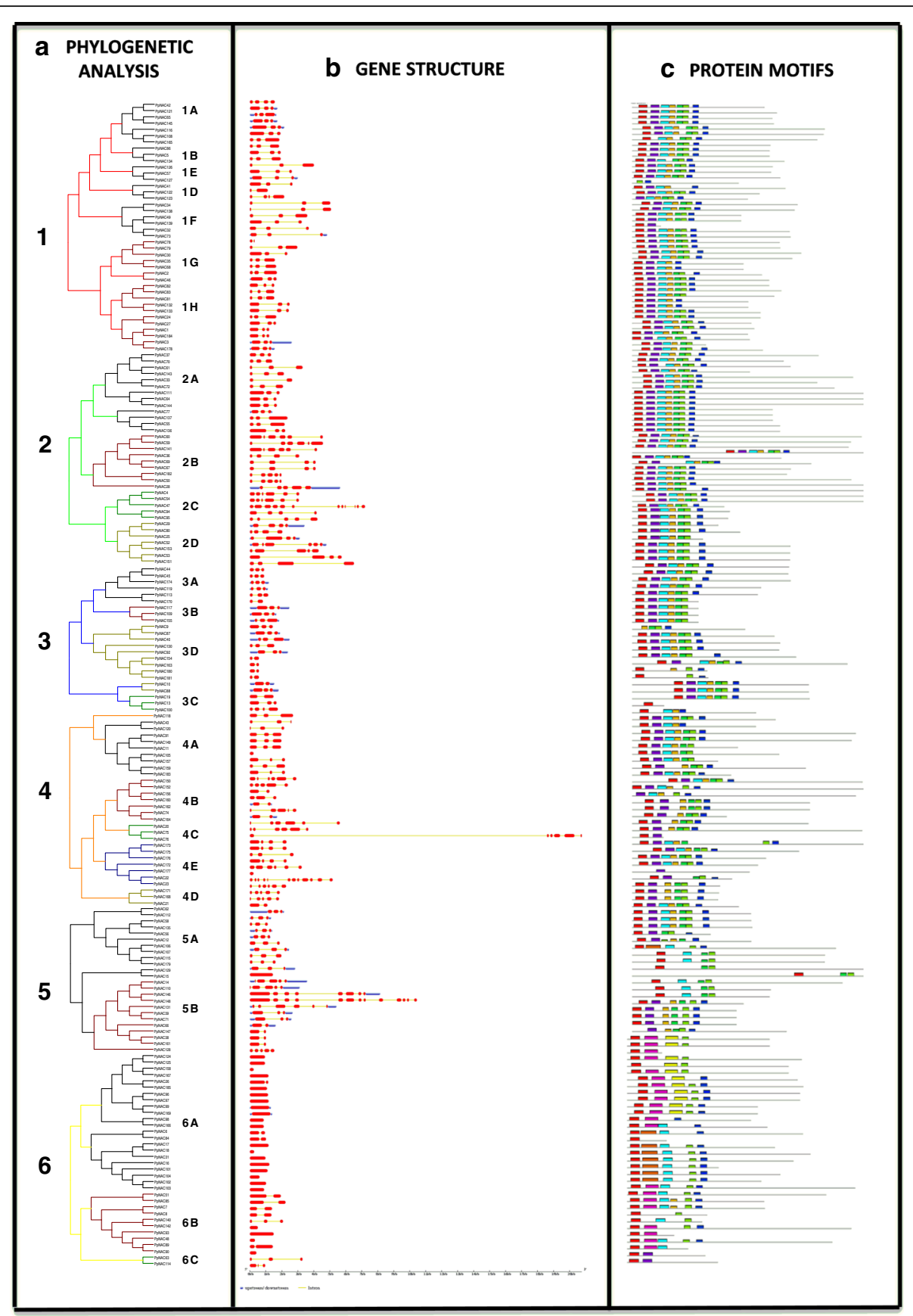

Fig. 4 Phylogenetic tree of all 6 groups of PpNAC transcription factors, their gene structures and protein motifs: a, Phylogenetic tree: The amino acid sequences of all 6 groups were aligned using MUSCLE, and the phylogenetic tree was constructed using the neighbor-joining method through MEGA7 software. Groups are shown by numbers from 1 to 6 with red, green, blue, orange, black and yellow color trees while their subgroups are showed with alphabet letters. $\mathbf{b}$, Gene structure: The exons are represented by red boxes while introns with yellow lines and blue bars indicate the upstream or downstream which was elucidated by using Gene Structure Display Server. The scale bar is represented $1.0 \mathrm{~kb}$ (middle). c, Protein motif: schematic diagrams of possible conserved motifs in PpNAC proteins, MEME tool was used to find out the conserved motifs. Conserved motifs (1-10) are indicated by different colored boxes while the non-conserved sequences are showed by the gray lines

might have some relation to fruit color developments in pear (Fig. 6).

(iii) We also examined the PpNAC genes from RPKM data during different fruit development stages in three pear species i.e. P. pashia, $P$. calleryana, and $P$. pyrifolia during $0,7,35$ and 85 days after flowering to fruit maturity with two replicates of each (Fig. 7a, b, c). Heat map representations according 


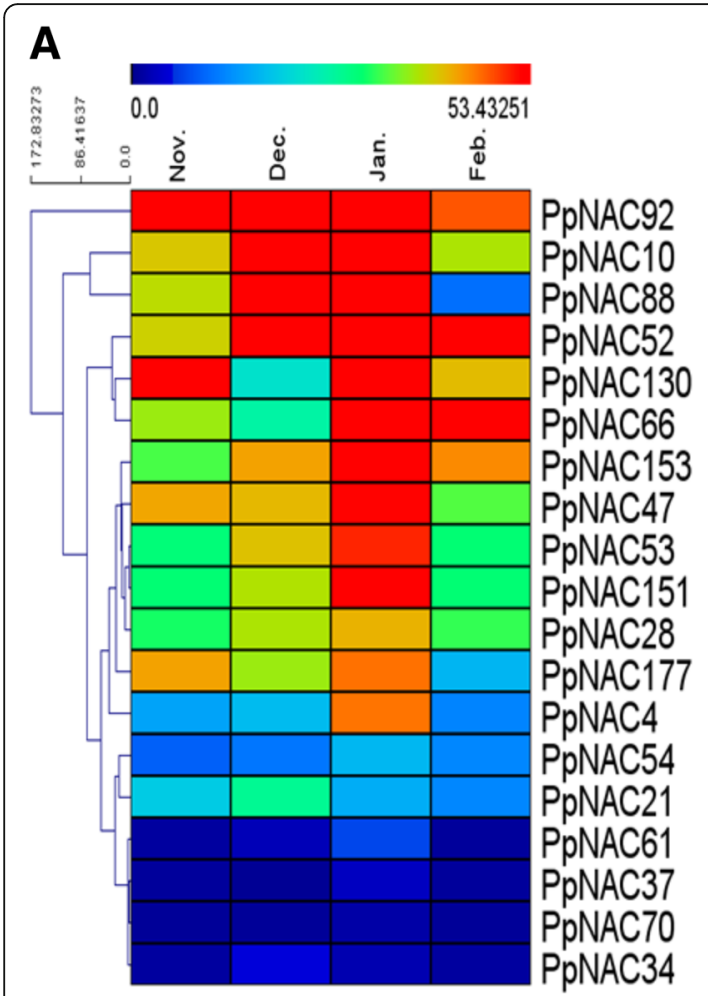

B
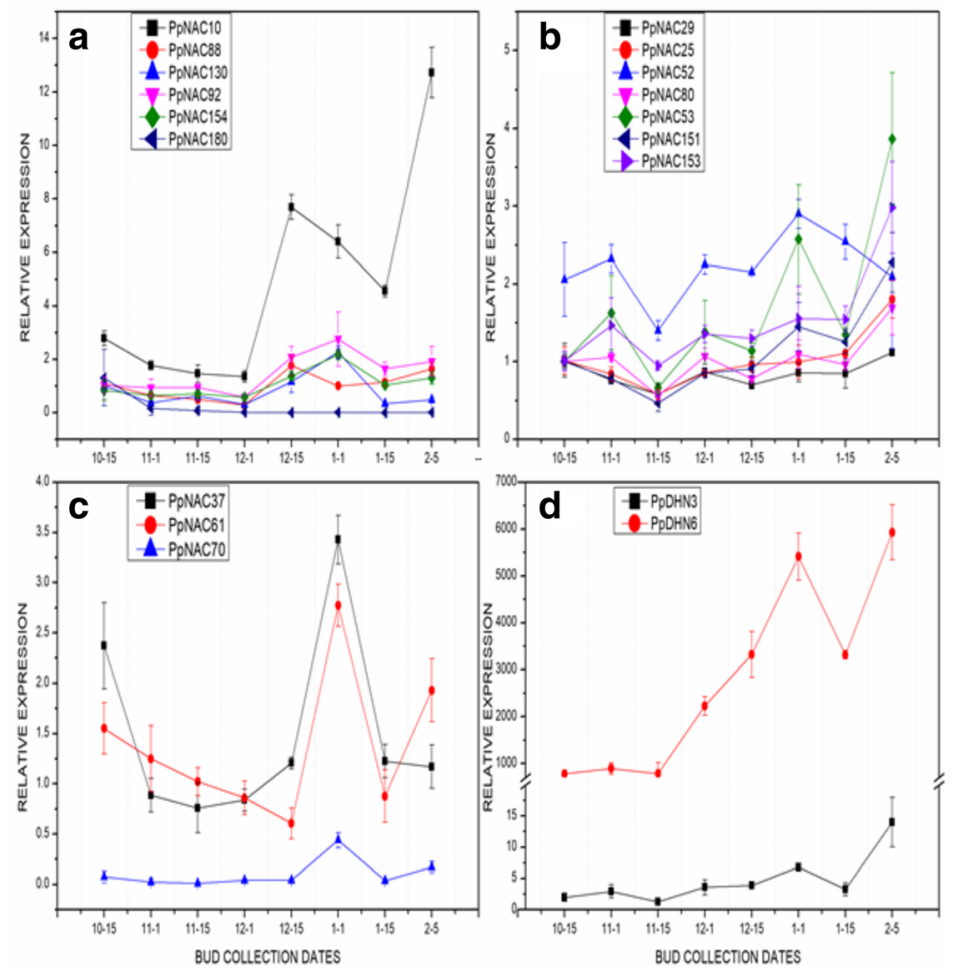

Fig. 5 Heat map presentation and expression analysis of selected PpNAC TFs during bud endodormancy in different pear cultivars. A, Heat map from RNA-seq data of differentially expressed PpNAC TFs during bud endodormancy period in P. pyrifolia 'Suli' during the entire endodormancy cycle. The color key at the top of heat map represents log2 of RPKM values. B, Expression analysis of the identified PpNAC genes during bud endodormancy in P. pyrifolia cv. Cuiguan. B-a, b, c, The expression patterns of selected genes from subgroups 3D, 2D and 2A in pear buds collected from Oct.-15 to Feb.-5 during the endodormancy period respectively. B-d, Endorsement of dormancy is assessed by the expressions of dehydrin genes with same samples. The data were normalized to the pear ACTIN expression level and the mean expression value was premeditated from 4 independent replicates while the standard deviation are also shown by vertical bars

to group and subgroup distributions demonstrated that subgroups $1 \mathrm{E} \& 4 \mathrm{C}, 1 \mathrm{G} \& 4 \mathrm{E}$ and $1 \mathrm{~F} \& 5 \mathrm{~A}$ played pivotal roles during early, middle and late fruit developments respectively in pear

(Additional file 6). From the detailed evaluation, we found 22 differentially expressed genes that showed period specific expressions during fruit developments with minor differences among all species (Fig. 7a, b, c). Among them 5 PpNACs (1E, $2 \mathrm{C}, 4 \mathrm{C}$ and $4 \mathrm{D})$ belonged to early, 7 (1G, $1 \mathrm{D}$ and $4 \mathrm{E})$ associated to middle, $8(1 \mathrm{~F}$ and $5 \mathrm{~A})$ related to late fruit developments in pear while 2 (6A and 6B) showed higher expressions during fruit maturity in all species. Because we had no plant material during fruit developmental stages, so, from graphical representation based on RNA-seq values, we concluded that within differential expressed genes, PpNAC 127 (1E), PpNAC 46 (1G) and PpNAC 56 (5A) were most probably related to early, middle and late fruit developments respectively in all pear species (Fig. 7d).

\section{Expression profiles of PpNAC genes during stress tolerance pathways}

Besides, NAC transcription factors had also been implicated in regulating the tolerances against different abiotic stresses in pear. Because we had no transcriptomic data of pear related to different abiotic stresses, so to find out the possible $P p N A C$ genes related to different stresses, we passed through some membrane-associated NAC proteins including NTLs of Arabidopsis (NTM1, NTM2, and NTL1-10) and many stress-responsive marker genes (e.g., ATAF1/2, ANAC019) in subgroups 2D and 3D through smart blast (Table 1). Study of MdNAC genes in apple during stress conditions also helped us to reconfirm the subset of $M d N A C$ genes ( $M d N A C 33$, $M d N A C 143$, and MdNAC168) belonged to subgroup $3 \mathrm{D}$ and indicated a high level of sequence resemblances with the stress-related marker genes. These findings enforced us to check the subgroups $2 \mathrm{D}$ and $3 \mathrm{D}$ that they might have roles during stress conditions in pear. So, we started to give low temperature $\left(4{ }^{\circ} \mathrm{C}\right)$, drought (15\% PEG 6000) and high salinity (200 mM NaCl) 

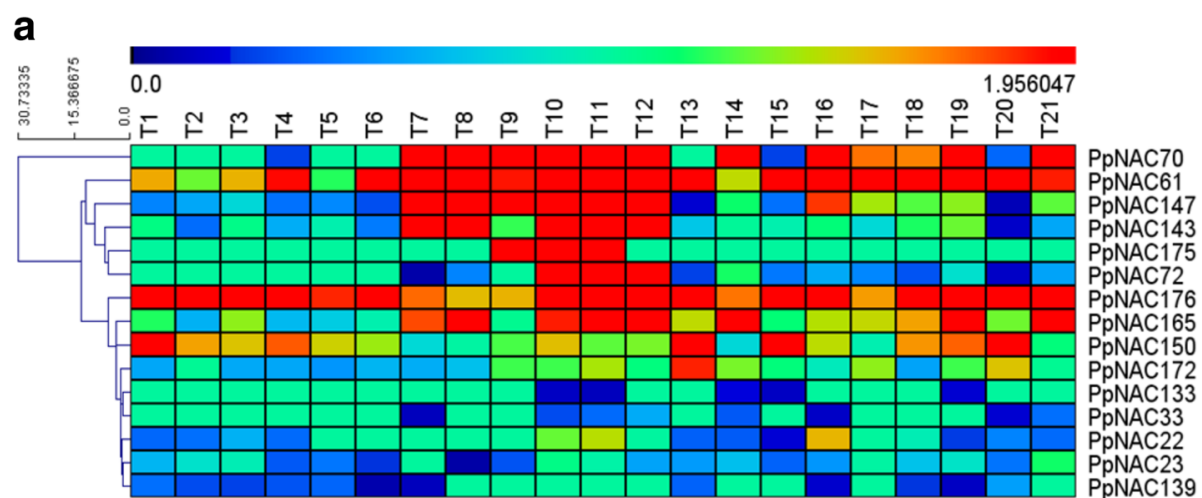

b
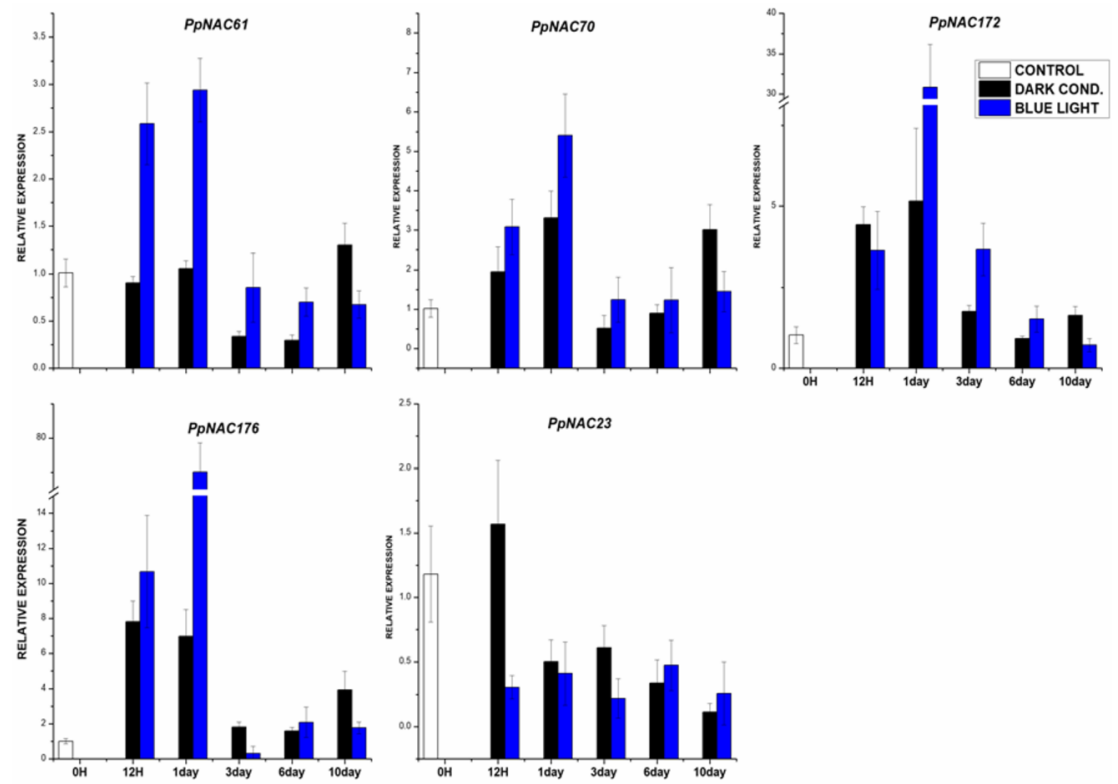

Fig. 6 Heat map presentation and expression analysis of selected PpNAC TFs during fruit color development in different pear cultivars. a, Heat map of PpNAC TFs during fruit color development under sun light and dark light treatments i.e. T1-T3, T4-T6, T7-T9 and T10-T12 indicates 0 h, 6 h, 1 day and 6 days under sun light conditions while T13-T15, T16-T18 and T19 to T21 represents 6 h, 1 day and 6 days treatments under dark conditions respectively with three independent biological replicates of Pyrus pyrifolia 'Meirensu'. The color key at the top of heat map represents $\log 2$ of RPKM values. b, Expression analysis of the identified PpNAC genes during fruit color development in P. pyrifolia 'Red Zaosu'. Expression representation of some genes from subgroup 2A (PpNAC61, PpNAC70) and 4E (PpNAC172, PpNAC176, PpNAC23) under blue light and dark treatments with 0 h, 12 h, 1 day, 3 days, 6 days and 10 days interval. The data were normalized to the pear ACTIN expression level and the mean expression value was premeditated from 4 independent replicates while the standard deviation are also shown by vertical bars

stresses for $0 \mathrm{~h}, 1 \mathrm{~h}, 6 \mathrm{~h}, 12 \mathrm{~h}$ and $48 \mathrm{~h}$ to explants of $P$. pyrifolia 'Suli' to check the expressions of above subgroups. qPCR results demonstrated that all members of subgroups $2 \mathrm{D}$ and $3 \mathrm{D}$ had high expressions during low temperature, salt and drought stresses as compared to control (Fig. 8). In detail, PpNACs 154E180, PpNACs 25E88, PpNACs 130E92 might have strong relations with freezing, salt and drought tolerances in pear plants respectively (Fig. 8a \& b). To endorse the NAC relation and stress status of all explants, we checked the expressions of stress responsive genes in same samples and made a predicted decision that PpCOR47E15A,PpRD29AEKIN and $P p D H N 3 \mathcal{E} 6$ might had relation with above stress related
$P p N A C$ genes during freezing, salt and drought tolerances respectively (Fig. 8c).

\section{Discussion}

We found 185 NAC genes containing full ORFs in Pyrus pyrifolia White Pear Group through genome-wide analysis (Table 1). This TF seemed to be one of the largest compared with other plant species as per findings till now 117 NAC genes in Arabidopsis [3], 151 in Rice [28], 79 in grape [29], 152 in tobacco and soybean [30, 31] and 180 in Apple [39]. The largest numbers indicated that NAC genes had expanded with evolution $[2,9]$ in pear. It is relatively limited to use Arabidopsis NAC 


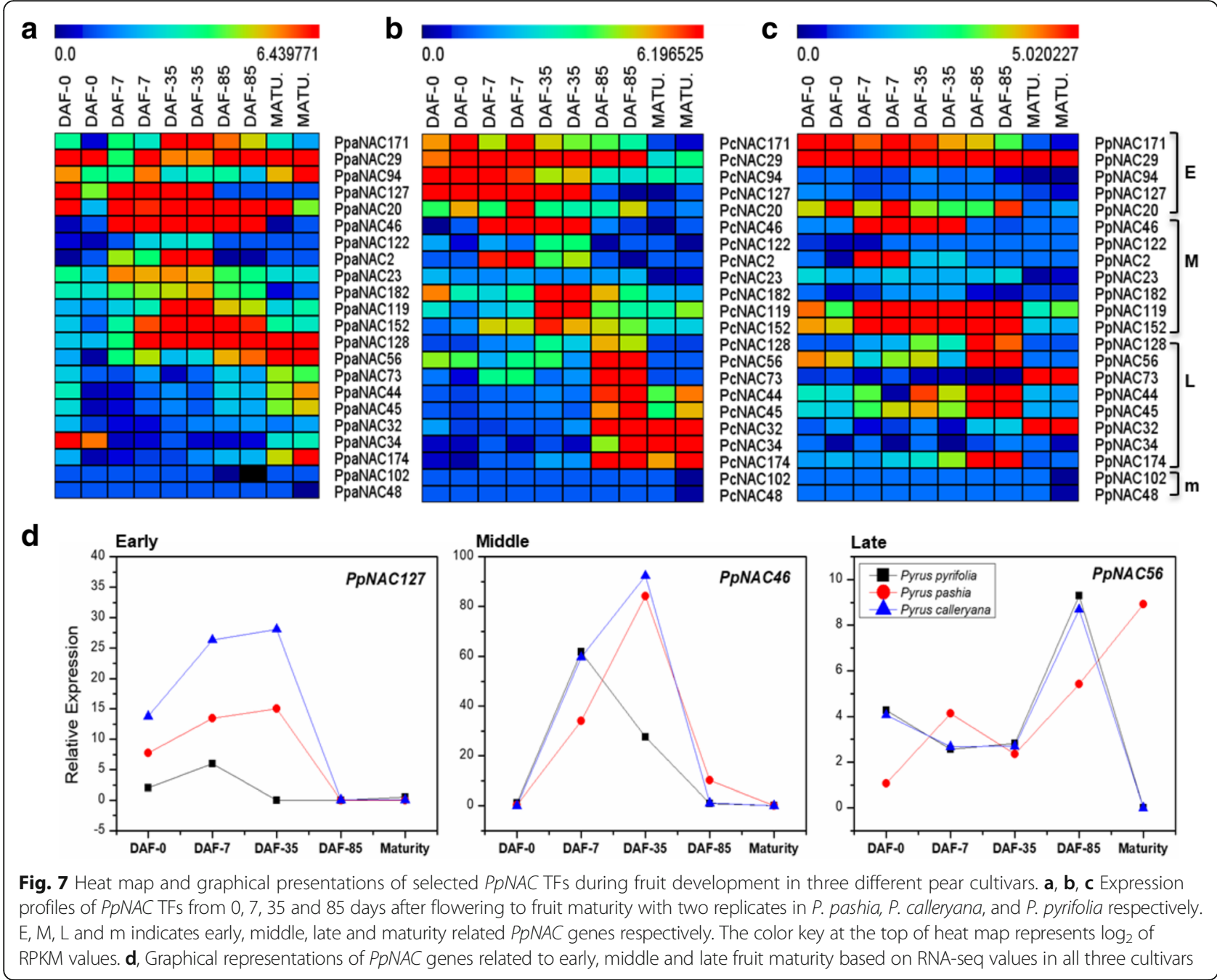

genes in phylogenetic tree as queries so to examine the evolutionary history, classification and functional prediction; we constructed a phylogenetic analysis of pear (185), apple (180) and Arabidopsis (138). Based on the full length sequences of NAC proteins, phylogenetic tree split the PpNAC genes into 6 distinct groups as previously found in Apple [32]. PpNACs, MdNACs and AtNACs genes from groups 1, 2, 3 and 5 indicated that these genes were not only homologous but might also be evolved from a common ancestor during evolution. In contrary, NACs from groups 4 and 6 implied that Rosaceae crops had common ancestors but different from Arabidopsis (Fig. 1).

Whole-genome duplication (WGD) event must had happened in pear about 30-45 MYA in prior to divergence with apple [34] and this gene duplications played vital roles in expansions, rearrangements and functional variations among NAC genes. So, collinerity of PpNAC genes and this WGD might have driven the expansions of this superfamily in pear and resulted in duplications of 44 PpNAC gene pairs. These duplications were observed in all groups but groups 4 and 6 showed only 2 and 1 pair of duplications respectively (Fig. 3). MEME showed that groups 1-5 had similar motifs (Motif 1-Motif 7) with minor changes and exchanges but in contrast, members of group 6 also showed some additional motifs (Motif 8, 9, and 10) (Fig. 4b). Gene structures also displayed no intronic regions in mostly members of group 6 (Fig. 4c). So, independent evolution, less duplication, different motifs and no intronic regions demonstrated that genes from groups 6 and 4 had functions of complicated transcriptional regulations only in woody perennial species and the members from Arabidopsis might have been lost in Rosaceae crops following the divergence during the evolutionary history. Our findings also demonstrated that genes in the same subgroups shared the similar gene structures and motifs and might have the similar gene functions (Fig. 4). Therefore, we did comparison with known functions of NAC genes in Arabidopsis and predicted the functions of candidate $P p N A C$ genes according to 


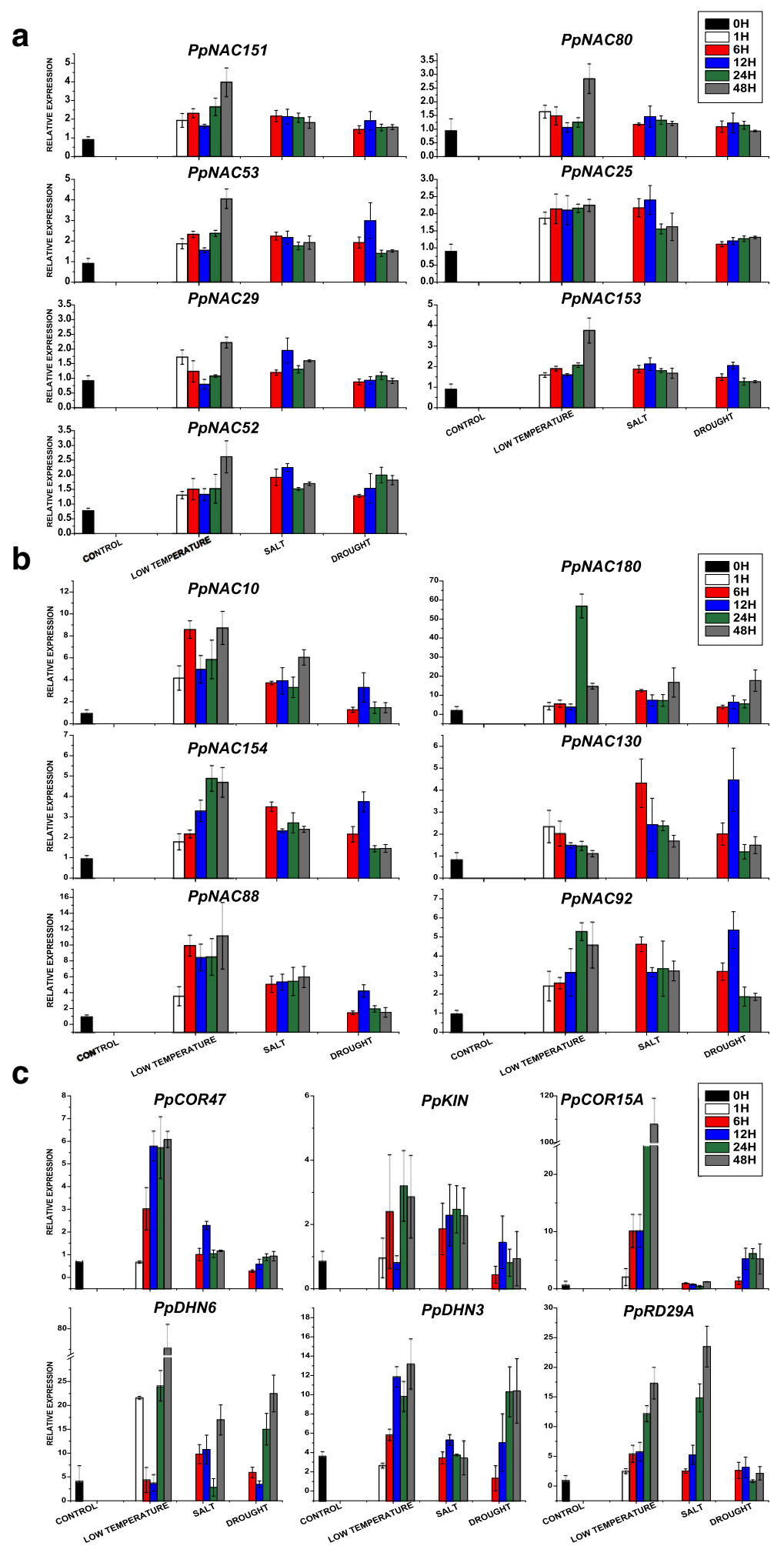

Fig. 8 Expression evaluation of the identified PpNAC and stress related genes under different abiotic stresses in P. pyrifolia 'Suli'. a, b qRT-PCR was used to examine the expression patterns of subgroups $2 \mathrm{D}$ and $3 \mathrm{D}$ in pear explants under low temperature $\left(4^{\circ} \mathrm{C}\right)$, drought (15\% PEG 6000) and high salinity (200 mM $\mathrm{NaCl})$ stresses for $0 \mathrm{~h}, 1 \mathrm{~h}, 6 \mathrm{~h}, 12 \mathrm{~h}$ and $48 \mathrm{~h}$ represented by black, white, red, blue, green and gray bars in graphs respectively. c, To reconfirm the stress status and NAC relation during abiotic stresses, different stress responsive and COR genes are analyzed in same samples. The data were normalized to the pear ACTIN expression level and the mean expression value was premeditated from 4 independent replicates while the standard deviation are shown by vertical bars 
best smart hit. As previous study reported that most of the subgroups in Arabidopsis were functioned in plant growth [40], signaling $[7,41]$ and stress response [42, 43] so, we also predict the same functions of these subgroups in pear except groups 4 and 6 (Table 1).

Gene expression is one of the basic evidence for gene functions. To find out the predicted functions of PpNACs, three different sets of RNA-Seq data provided us complete expression profiles of this superfamily during bud dormancy, fruit color and developments (Additional files 4, 5 and 6). Previously proved that PbeNAC1 functioned in cold tolerance by interacting with bud dormancy and cold related DREBs and DHN genes [25, 44-46]. So, we found 9 PpNAC TFs (PpNAC 37, 61, 70, 53, 151, 10, 92, 130 and 154) and also 2 DHNs genes (PpDHN3 and PpDHN6) that might be related to winter dormancy in pear (Fig. 5) because previously study on apple demonstrated that the reduction of free water content in dormant buds coincides with DHN protein accumulation during winter [39]. Regarding the role of NAC genes in anthocyanin accumulation and color development, PpeNAC1 caused anthocyanin pigmentation in overexpressed transgenic tobacco [16], AtNAC078 regulated the flavonoid biosynthesis under high-light in Arabidopsis [47] and in our previous study, an involvement of blue light was also found in anthocyanin accumulation in 'Red Zaosu' pear [36, 38]. Through RPKM data analysis and the homologous genes of PpeNAC1 in pear, we found 15 PpNAC genes that might be related to light and anthocyanin accumulation (Fig. 6a) from which 5 PpNAC genes (PpNACs $61,70,172,176$ and 23 ) possibly associated to blue light induced pigmentation in 'Red Zaosu' pear (Fig. 6b). Different fruit development stages in P. calleryana, $P$. pashia and $P$. pyrifolia revealed 22 differentially expressed genes mostly from groups 1 and 5 that might be related to fruit developments in pear (Fig. 6 and Additional file 4) while in apple 13 out of $182 \mathrm{MdNAC}$ genes were found to be differentially expressed during the stages of fruit growth and maturity [48]. Similarly, predicted functions of groups 1 and 5 were also related to plant growth and development such as cell expansion [40], root initiation [49], vascular tissue development and secondary cell wall formation in Arabidopsis [50] (Table 1).

Due to the presence of stress-responsive marker genes in subgroups 2D and 3D, we found 13 PpNAC genes from these subgroups which were related to abiotic stresses in pear (Fig. 8) [32, 42] and previously $17 M d N A C$ genes were reported in apple that responded to one or more types of abiotic stresses [32]. Further, the expression levels of stress responsive genes (NtRD29A, $N t R D 1, N t E R D 10 D, N t L E A 5$, NtNCED1 etc) were higher in PbeNAC1 transgenic tobacco plants as compared to
WT under drought and low temperature stresses [25] which indicated that a transcription activator had an ability to activate a series of target genes that helped in adaptations and encountered these abiotic stresses [51, 52]. Likewise, in our study we also found highly expressed stress responsive genes PpCOR47\&15A, PpRD29AEKIN and $P p D H N 3 \mathcal{E} 6$ that might had relation with this transcription activator " $P p N A C$ " during freezing, salt and drought tolerances.

NAC TFs have received much attention during different biological pathways of plant growth \& developments and biotic \& abiotic stresses [53, 54]. Accordingly, our findings indicated that $P p N A C \mathrm{TFs}$ not only played major roles during a number of biological processes of plant development like fruit development and maturity, anthocyanin accumulation and growth cessation but also during stress tolerance pathways like freezing, salt and drought in pear.

\section{Conclusions}

Our work aimed to find the evolution, gene functions and expression patterns of NAC gene family in pear. Whole genome duplication (WGD) and duplication events played their important roles in expansion of this gene family in pear. Functional predications and expression divergences among different groups of $P p N A C$ genes showed their involvement in multiple regulation pathways in pear. Multiple evidences supported that 9, 5 and $22 P p N A C$ genes belonged to bud endodormancy, fruit pigmentation and fruit developments respectively while 13 genes from subgroups $2 \mathrm{D}$ and $3 \mathrm{D}$ might be involved in responses to abiotic stresses in pear. Hence, these results of PpNAC TFs will help to build a solid foundation for the future exploration and potential improvements in the stress resistance, growth and development of pear as well as other crops through genetic engineering approaches.

\section{Methods \\ Identifications of NAC TFs in pear}

The predicted protein sequences to identify the members of NAC gene family were retrieved from the pear genome project (http://peargenome.njau.edu.cn/) [34]. First, a Hidden Markov Model search (HMM search) was done by using the pfam \# PF01849 which contained PpNAC domains from domain analysis programs (http://pfam.xfam.org/). The other search was from Plant Transcription factor database (http://planttfdb.cbi.pku.edu.cn) of Pear NAC TFs. The sequences which were redundant, incomplete and high e-value had been rejected. Further, we used the SMART tool (http://smart.embl-heidelberg.de/) and the InterProScan tool (http://www.ebi.ac.uk/Tools/pfa/ iprscan/) to find the presence of NAM domain in all selected NAC genes. The NAC TFs in Arabidopsis were 
downloaded from The Arabidopsis Information Resource (https://www.arabidopsis.org/) while for apple, NAC TFs were downloaded from GDR database (Genome Database for Rosaceae: http://www.rosaceae.org/) and Apple GFDB database (Apple Gene Function and Gene Family Database: http://apple.sdnx.co/) following the previous study on $M d N A C$ TFs [32].

\section{Chromosome location and gene duplication of PpNAC genes}

Chromosome locations of pear NAC genes were analyzed using BLAST software 2.25 (ftp:/ncbi.nlm.nih.gro/ blast/executables/release/) to align the $N A C$ sequences against the pear genome sequence and got the chromosome positions of all NAC genes [55]. The length of each chromosome was also obtained from pear genome database. The data were integrated and plotted by using Mapchart software (v.2.2) [56].

Collinearity blocks of whole genome and NAC TFs within the species were identified by using MCScanX algorithm with default settings and followed the given instructions on user manual with E-value $\leq 1 \mathrm{e}-10$ [57].

\section{Sequence alignment and phylogenetic analysis of PpNAC TFs}

The sequences of pear, apple and Arabidopsis were aligned by using the MUSCLE program [58] while the phylogenetic trees for the PpNAC, MdNAC and AtNAC protein sequences were constructed by using the maximum likelihood method of the MEGA7.0 (Molecular Evolutionary Genetics Analysis) program [58]. The reliability of the trees was also tested using a bootstrapping method and the images of the phylogenetic trees were drawn.

\section{Gene structure and motif analyses}

CDS sequences of each PpNAC gene with their corresponding full-length genomic sequences were uploaded on online website: Gene Structure Display Server (GSDS) (http://gsds.cbi.pku.edu.cn/) and the gene structures of the PpNAC gene family were obtained and downloaded. Moreover, conserved motifs were perceived in pear NAC family members using the motif analysis tool MEME (http://meme-suite.org/tools/meme) version 4.12.0 with the default parameters except for two: any number of repetitions; maximum number of motifs, 10 .

\section{Plant material and RPKM data analysis}

For stress treatments, vegetative buds of 'Suli' pear (Pyrus pyrifolia white pear group) were collected before bud break in March 2018. After bud sterilization and washing they were grown in $1 / 2 \mathrm{MS}$ media and put in tissue culture room for getting pear seedlings. When the seedlings were uniform in size and at 6 to 8 leaves stage then they were randomly selected for stress treatments. For low temperature, seedlings were put at $4{ }^{\circ} \mathrm{C}$ while during drought and salt stresses, 15\% PEG 6000 and $200 \mathrm{mM} \mathrm{NaCl}$ were added into 1/2 MS media respectively. Each sample with three replicates were collected after $0 \mathrm{~h}, 1 \mathrm{~h}, 6 \mathrm{~h}, 12 \mathrm{~h}$ and $48 \mathrm{~h}$ and immediately frozen in liquid nitrogen and RNA extracted without storage. The plant materials for coloration [38] and bud endodormancy [55] are the same as recently published.

Three different RNA-seq datasets i.e. pear bud endodormancy, fruit coloration and different fruit development stages were already been published [35-37]. MultiExperiment Viewer (v 4.8.1) software (www.tm4.org) was used to make the heat map of RNA-Seq data followed the given instructions on $\mathrm{MeV}$ quickstart guide.

\section{RNA extraction and cDNA synthesis}

Total RNA was extracted from each sample using a modified cetyltrimethylammonium bromide (CTAB) method [59]. Genomic DNA was eliminated with DNase I and the concentration of total RNA was measured. First-strand cDNA was synthesized from $4 \mu \mathrm{g}$ DNA-free RNA using the iScript cDNA Synthesis Kit (Bio-Rad, CA, USA) following the manufacturer's instructions. The cDNA was diluted 10-fold and used as the template for real-time quantitative PCR (qRT-PCR) analysis.

\section{Real-time quantitative RT-PCR analysis}

The solution of qRT-PCR reaction ( $15 \mu \mathrm{L}$ total volume) was composed of $7.5 \mu \mathrm{L}$ SYBR Premix Ex Taq ${ }^{\text {TM }}$ (Tli RNaseH Plus), $1 \mu \mathrm{L}$ of cDNA (corresponding to $0.5 \mu \mathrm{g}$ total RNA), $0.5 \mu \mathrm{L}$ from each forward and reverse primers $(10 \mu \mathrm{M})$, and $5.5 \mu \mathrm{L}$ RNase-free water. Following program: $30 \mathrm{~s}$ at $95{ }^{\circ} \mathrm{C}$ followed by 40 cycles of $95{ }^{\circ} \mathrm{C}$ for $5 \mathrm{~s}$ and $60{ }^{\circ} \mathrm{C}$ for $20 \mathrm{~s}$ was run for the reaction in a CFX Connect ${ }^{\mathrm{Tm}}$ real-time PCR system (Bio-Rad, Hercules, CA, USA). Melting curves and sequencing of the qRT-PCR products confirmed the specificity of the qRT-PCR primers. Gene transcript levels were measured as $2^{-\Delta \Delta C t}$ and normalized against PpActin (JN684184) transcript levels [35]. Data were subjected to one-way ANOVA while the Tukey's range test $(P<0.05)$ was used to measure means among different treatments in all samples. Primer3 program (http://bioinfo.ut.ee/primer3-0.4.0/) is used to design the primers which are provided in Additional file 7.

\section{Additional files}

Additional file 1: Detailed information, group wise distribution and protein sequences of selected pear NAC TFs. (XLSX 53 kb)

Additional file 2: Phylogenetic tree of apple, pear and Arabidopsis (DOCX $350 \mathrm{~kb}$ )

Additional file 3: Frequency Distribution of PpNAC genes on each chromosome. (DOCX 117 kb) 
Additional file 4: Heat map presentation of all PpNAC TFs according to group distributions during bud endodormancy in P. pyrifolia Chinese 'Suli' (DOCX $144 \mathrm{~kb}$ )

Additional file 5: Heat map presentation of all PpNAC TFs according to group wise distributions during fruit color development in P. pyrifolia 'Meirensu' (DOCX 242 kb)

Additional file 6: Heat map presentation of all PpNAC TFs according to group distributions during fruit developments in three pear cultivars viz. P. calleryana, P. pashia and P. pyrifolia. (DOCX $925 \mathrm{~kb}$ )

Additional file 7: All primer sequences used for qRT-PCR analysis (DOCX $138 \mathrm{~kb}$ )

\section{Abbreviations}

BLAST: The Basic Local Alignment Search Tool; HMM: Hidden Markov Model; MEGA: Molecular Evolutionary Genetics Analysis; MEME: Multiple Em for Motif Elicitation; MUSCEL: Multiple Sequence Comparison by Log-Expectation; ORF: Open reading frame; Pfam: Protein family; RNA-seq: Ribose nucleic acid sequencing; RPKM: Reads per kilobase per million reads; SMART: A Simple Modular Architecture Research Tool; TF: Transcription factor

\section{Acknowledgments}

We thank Mrs. Junbei Ni and Dr. Xiaoyan Yue for the provision of RNA-Seq data. We also thank Mr. Ruiyan Tao for providing the plant materials related to fruit color developments in pear.

\section{Funding}

This work was supported by the National Natural Science Foundation of China (31772272, 31501736 and 31471852), the Fundamental Research Funds for the Central Universities (2018QNA6022),and the Earmarked Fund for China Agriculture Research System (CARS-28).

\section{Availability of data and materials}

All sequence information on pear is available at the pear genome database (http://peargenome.njau.edu.cn/). All Arabidopsis protein sequences were downloaded from The Arabidopsis Information Resource (TAIR) (https:/ www.arabidopsis.org). All apple proteins were downloaded from GDR database (Genome Database for Rosaceae: http://www.rosaceae.org/) and Apple GFDB database (Apple Gene Function and Gene Family Database: http://apple.sdnx.co/). Transcriptome sequencing (RNA-Seq) data of pear bud endodormancy, fruit coloration and different fruit development stages have already been published [35-37]. All data generated or analyzed during this study are included in this article and its supplementary information files.

\section{Authors' contributions}

SB and YT perceived and planned the study and MA performed most of the experiments and analysis. MA, XY and QY helped collected the samples and extracted total RNAs for qPCR. JL and WJ helped in run bioinformatics software and data arrangement. MA, SB and Y.T wrote the manuscript. All authors read and approved the final manuscript.

\section{Ethics approval and consent to participate}

Not applicable.

\section{Consent for publication}

Not applicable.

\section{Competing interests}

The authors declare that they have no competing interest.

\section{Publisher's Note}

Springer Nature remains neutral with regard to jurisdictional claims in published maps and institutional affiliations.

\section{Author details}

'Department of Horticulture, Zhejiang University, Hangzhou 310058, Zhejiang, China. ${ }^{2}$ The Key Laboratory of Horticultural Plant Growth, Development and Quality Improvement, the Ministry of Agriculture of China, Hangzhou 310058, Zhejiang, China. ${ }^{3}$ Zhejiang Provincial Key Laboratory of Integrative Biology of Horticultural Plants, Zhejiang 310058, Hangzhou, China.
Received: 22 June 2018 Accepted: 16 September 2018

Published online: 03 October 2018

\section{References}

1. Souer E, van Houwelingen A, Kloos D, Mol J, Koes R. The no apical meristem gene of Petunia is required for pattern formation in embryos and flowers and is expressed at meristem and primordia boundaries. Cell. 1996;85(2): 159-70.

2. Puranik S, Sahu PP, Srivastava PS, Prasad M. NAC proteins: regulation and role in stress tolerance. Trends Plant Sci. 2012;17(6):369-81.

3. Ooka H, Satoh K, Doi K, Nagata T, Otomo Y, Murakami K, Matsubara K, Osato $\mathrm{N}$, Kawai J, Carninci P. Comprehensive analysis of NAC family genes in Oryza sativa and Arabidopsis thaliana. DNA Res. 2003;10(6):239-47.

4. Delessert C, Kazan K, Wilson IW, Straeten DVD, Manners J, Dennis ES, Dolferus R. The transcription factor ATAF2 represses the expression of pathogenesis-related genes in Arabidopsis. Plant J. 2005;43(5):745-57.

5. Puranik S, Bahadur RP, Srivastava PS, Prasad M. Molecular cloning and characterization of a membrane associated NAC family gene, SiNAC from foxtail millet [Setaria italica Setaria (L.) P. Beauv.]. Mol Biotechnol. 2011;49(2): $138-50$.

6. Tran LS, Nakashima K, Sakuma Y, Simpson SD, Fujita Y, Maruyama K, Fujita M, Seki M, Shinozaki K, Yamaguchi SK. Isolation and functional analysis of Arabidopsis stress-inducible NAC transcription factors that bind to a drought-responsive cis-element in the early responsive to dehydration stress 1 promoter. Plant Cell. 2004;16(9):2481-98.

7. Kim SG, Kim SY, Park CM. A membrane-associated NAC transcription factor regulates salt-responsive flowering via FLOWERING LOCUS T in Arabidopsis. Planta. 2007;226(3):647-54.

8. Hibara K, Takada S, Tasaka M. CUC1 gene activates the expression of SAMrelated genes to induce adventitious shoot formation. Plant J. 2003;36(5): 687-96.

9. Duval M, Hsieh TF, Kim SY, Thomas TL. Molecular characterization of AtNAM: a member of the Arabidopsis NAC domain superfamily. Plant Mol Biol. 2002; 50(2):237-48.

10. Sperotto RA, Ricachenevsky FK, Duarte GL, Boff T, Lopes KL, Sperb ER, Grusak MA, Fett JP. Identification of up-regulated genes in flag leaves during rice grain filling and characterization of OsNAC5, a new ABAdependent transcription factor. Planta. 2009;230(5):985-1002.

11. Shu K, Liu XD, Xie Q, He ZH. Two faces of one seed: hormonal regulation of dormancy and germination. Mol Plant. 2016;9(1):34-45.

12. Fujita M, Fujita Y, Maruyama K, Seki M, Hiratsu K, Ohme TM, Tran LP, Yamaguchi S, Shinozaki K. A dehydration-induced NAC protein, RD26, is involved in a novel ABA-dependent stress-signaling pathway. Plant J. 2004; 39(6):863-76.

13. Kim YS, Kim SG, Park JE, Park HY, Lim MH, Chua NH, Park CM. A membranebound NAC transcription factor regulates cell division in Arabidopsis. Plant Cell. 2006;18(11):3132-44.

14. Li Q, Lin YC, Sun YH, Song J, Chen H, Zhang XH, Sederoff RR, Chiang VL. Splice variant of the SND1 transcription factor is a dominant negative of SND1 members and their regulation in Populus trichocarpa. Proc Natl Acad Sci U S A. 2012;109(36):14699-704.

15. Giovannoni JJ. Genetic regulation of fruit development and ripening. Plant Cell. 2004;16:170-80.

16. Zhou H, Lin WK, Wang H, Gu C, Dare AP, Espley RV, He H, Allan AC, Han Y. Molecular genetics of blood-fleshed peach reveals activation of anthocyanin biosynthesis by NAC transcription factors. Plant J. 2015;82(1):105-21.

17. Wu Y, Deng Z, Lai J, Zhang Y, Yang C, Yin B, Zhao Q, Zhang L, Li Y, Yang C. Dual function of Arabidopsis ATAF1 in abiotic and biotic stress responses. Cell Res. 2009;19(11):1279.

18. Nakashima K, Tran LSP, Van Nguyen D, Fujita M, Maruyama K, Todaka D, Ito Y, Hayashi N, Shinozaki K, Yamaguchi-Shinozaki K. Functional analysis of a NAC-type transcription factor OsNAC6 involved in abiotic and biotic stressresponsive gene expression in rice. Plant J. 2007;51(4):617-30.

19. Collinge M, Boller T. Differential induction of two potato genes, Stprxz and StNAC, in response to infection by Phytophthora infestans and to wounding. Plant Mol Biol. 2001;46(5):521-9.

20. Hegedus D, Yu M, Baldwin D, Gruber M, Sharpe A, Parkin I, Whitwill S, Lydiate D. Molecular characterization of Brassica napus NAC domain transcriptional activators induced in response to biotic and abiotic stress. Plant Mol Biol. 2003;53(3):383-97. 
21. Hu H, You J, Fang Y, Zhu X, Qi Z, Xiong L. Characterization of transcription factor gene SNAC2 conferring cold and salt tolerance in rice. Plant Mol Biol. 2008:67(1-2):169-81.

22. Kaneda T, Taga Y, Takai R, Iwano M, Matsui H, Takayama S, Isogai A, Che FS. The transcription factor OsNAC4 is a key positive regulator of plant hypersensitive cell death. EMBO J. 2009;28(7):926-36.

23. He XJ, Mu RL, Cao WH, Zhang ZG, Zhang JS, Chen SY. AtNAC2, a transcription factor downstream of ethylene and auxin signaling pathways, is involved in salt stress response and lateral root development. Plant J. 2005;44(6):903-16.

24. Boyer JS. Plant productivity and environment. Science. 1982;218(4571): 443-8.

25. Jin C, Li KQ, Xu XY, Zhang HP, Chen HX, Chen YH, Hao J, Wang Y, Huang $X S$, Zhang SL. A novel NAC transcription factor, PbeNAC1, of Pyrus betulifolia confers cold and drought tolerance via interacting with PbeDREBs and activating the expression of stress-responsive genes. Front Plant Sci. 2017;8: 1049.

26. An J, Li R, Qu F, You C, Wang X, Hao Y. An apple NAC transcription factor negatively regulates cold tolerance via CBF-dependent pathway. J Plant Physiol. 2018;221:74-80.

27. An J, Yao J, Xu R, You C, Wang X, Hao Y. An apple NAC transcription factor enhances salt stress tolerance by modulating the ethylene response. Physiol Plant. 2018; https://doi.org/10.1111/ppl.12724.

28. Nuruzzaman M, Manimekalai R, Sharoni AM, Satoh K, Kondoh H, Ooka H, Kikuchi S. Genome-wide analysis of NAC transcription factor family in rice. Gene. 2010;465(1-2):30-44.

29. Wang N, Zheng Y, Xin H, Fang L, Li S. Comprehensive analysis of NAC domain transcription factor gene family in Vitis vinifera. Plant Cell Rep. 2013; 32(1):61-75.

30. Han Q, Qiao P, Song Y, Zhang J. Structural analysis and tissue-specific expression patterns of a novel salt-inducible NAC transcription factor gene from Nicotiana tabacum cv. Xanthi. J Hortic Sci Biotechnol. 2014;89(6):700-6.

31. Pinheiro GL, Marques CS, Costa MD, Reis PA, Alves MS, Carvalho CM, Fietto $L G$, Fontes EP. Complete inventory of soybean NAC transcription factors: sequence conservation and expression analysis uncover their distinct roles in stress response. Gene. 2009:444(1):10-23.

32. Su H, Zhang S, Yuan X, Chen C, Wang XF, Hao YJ. Genome-wide analysis and identification of stress-responsive genes of the NAM-ATAF1,2-CUC2 transcription factor family in apple. Plant Physiol Biochem. 2013;71:11-21.

33. Borrill P, Harrington SA, Uauy C. Genome-wide sequence and expression analysis of the NAC transcription factor family in polyploid wheat. Genes, Genomes, Genet. 2017;7(9):3019-29.

34. Wu J, Wang Z, Shi Z, Zhang S, Ming R, Zhu S, Khan MA, Tao S, Korban SS, Wang $\mathrm{H}$. The genome of the pear (Pyrus bretschneideri Rehd.). Genome Res. 2013;23(2):396.

35. Liu G, Li W, Zheng P, Xu T, Chen L, Liu D, Hussain S, Teng Y. Transcriptomic analysis of 'Suli'pear (Pyrus pyrifolia white pear group) buds during the dormancy by RNA-Seq. BMC Genomics. 2012;13(1):700.

36. Bai S, Sun Y, Qian M, Yang F, Ni J, Tao R, Li L, Shu Q, Zhang D, Teng Y. Transcriptome analysis of bagging-treated red chinese sand pear peels reveals light-responsive pathway functions in anthocyanin accumulation. Sci Rep. 2017;7(1):63.

37. Ni J, Bai S, Gao L, Qian M, Zhong L, Teng Y. Identification, classification, and transcription profiles of the B-type response regulator family in pear. PLoS One. 2017;12(2):e0171523.

38. Tao R, Bai S, Ni J, Yang Q, Zhao Y, Teng Y. The blue light signal transduction pathway is involved in anthocyanin accumulation in 'Red Zaosu' pear. Planta. 2018:248(1):37-48.

39. Falavigna VDS, Porto DD, Buffon V, Margis PM, Pasquali G, Revers LF. Differential transcriptional profiles of dormancy-related genes in apple buds. Plant Mol Biol Report. 2013;32(4):796-813.

40. Pei H, Ma N, Tian J, Luo J, Chen J, Li J, Zheng Y, Chen X, Fei Z, Gao J. An NAC transcription factor controls ethylene-regulated cell expansion in flower petals. Plant Physiol. 2013;163(2):775-91.

41. Shahnejat BS, Tarkowska D, Sakuraba Y, Balazadeh S. Arabidopsis NAC transcription factor JUB1 regulates GA/BR metabolism and signalling. Nature Plants. 2016;2(3):16013

42. Kim SG, Lee AK, Yoon HK, Park CM. A membrane-bound NAC transcription factor NTL8 regulates gibberellic acid-mediated salt signaling in Arabidopsis seed germination. Plant J. 2008:55(1):77-88.
43. Li X, Li X, Li M, Yan Y, Liu X, Li L. Dual function of NAC072 in ABF3-mediated ABA-responsive gene regulation in Arabidopsis. Front Plant Sci. 2016;7:1075.

44. Niu Q, Li J, Cai D, Qian M, Jia H, Bai S, Sayed H, Liu G, Teng Y, Zheng X. Dormancy-associated MADS-box genes and microRNAs jointly control dormancy transition in pear (Pyrus pyrifolia white pear group) flower bud. J Exp Bot. 2016;67(1):239.

45. Hussain S, Niu Q, Qian M, Bai S, Teng Y. Genome-wide identification, characterization and expression analysis of the dehydrin gene family in Asian pear (Pyrus pyrifolia). Tree Gent Genome. 2015;11(5):110.

46. Barros PM, Gonçalves N, Saibo NJM, Oliveira MM. Cold acclimation and floral development in almond bud break: insights into the regulatory pathways. J Exp Bot. 2012;63(12):4585-96.

47. Morishita T, Kojima Y, Maruta T, Nishizawa YA, Yabuta Y, Shigeoka S Arabidopsis NAC transcription factor, ANAC078, regulates flavonoid biosynthesis under high-light. Plant Cell Physiol. 2009;50(12):2210-22.

48. Qijing Z, Tong L, Lijie Z, Wenxuan D, Aide W. Expression analysis of NAC genes during the growth and ripening of apples. Hortic Sci 2018;45(No. 1):1-10.

49. Kamiya M, Higashio SY, Isomoto A, Kim JM, Seki M, Miyashima S, Nakajima K. Control of root cap maturation and cell detachment by BEARSKIN transcription factors in Arabidopsis. Development. 2016;143(21):4063-72.

50. Yamaguchi M, Ohtani M, Mitsuda N, Kubo M, Ohme TM, Fukuda H, Demura T. VND-INTERACTING2, a NAC domain transcription factor, negatively regulates xylem vessel formation in Arabidopsis. Plant Cell. 2010;22(4):1249-63.

51. Li K, Xing C, Yao Z, Huang X. PbrMYB21, a novel MYB protein of Pyrus betulaefolia, functions in drought tolerance and modulates polyamine levels by regulating arginine decarboxylase gene. Plant Biotechnol J. 2017;15(9): 1186-203.

52. Huang $X$, Li K, Jin C, Zhang S. ICE1 of Pyrus ussuriensis functions in cold tolerance by enhancing PUDREBa transcriptional levels through interacting with PUHHP1. Sci Rep. 2015;5:17620.

53. Jensen MK, Kjaersgaard T, Nielsen MM, Galberg P, Petersen K, OShea C, Skriver K. The Arabidopsis thaliana NAC transcription factor family: structurefunction relationships and determinants of ANAC019 stress signalling. Biochem J. 2010;426(2):183-96.

54. Lu P, Chen N, An R, Su Z, Qi B, Ren F, Chen J, Wang X. A novel droughtinducible gene, ATAF1, encodes a NAC family protein that negatively regulates the expression of stress-responsive genes in Arabidopsis. Plant Mol Biol. 2007;63(2):289-305.

55. Yang Q, Niu Q, Li J, Zheng X, Ma Y, Bai S, Teng Y. PpHB22, a member of HDzip proteins, activates PpDAM1 to regulate bud dormancy transition in 'Suli'pear (Pyrus pyrifolia white pear group). Plant Physiol Biochem. 2018;127: 355-65.

56. Voorrips R. MapChart: software for the graphical presentation of linkage maps and QTLs. J Hered. 2002;93(1):77-8.

57. Wang Y, Tang H, DeBarry JD, Tan X, Li J, Wang X, Lee T, Jin H, Marler B, Guo H. MCScanX: a toolkit for detection and evolutionary analysis of gene synteny and collinearity. Nucleic Acids Res. 2012;40(7):49-50.

58. Kumar S, Stecher G, Tamura K. MEGA7: molecular evolutionary genetics analysis version 7.0 for bigger datasets. Mol Biol Evol. 2016;33(7):1870-4.

59. Zhang D, Yu B, Bai J, Qian M, Shu Q, Su J, Teng Y. Effects of high temperatures on UV-B/visible irradiation induced postharvest anthocyanin accumulation in 'Yunhongli no. 1'(Pyrus pyrifolia Nakai) pears. Sci Hortic. 2012;134:53-9.

60. Motte H, Verstraeten I, Werbrouck S, Geelen D. CUC2 as an early marker for regeneration competence in Arabidopsis root explants. J Plant Physiol. 2011; 168(13):1598-601

61. Mandaokar A, Thines B, Shin B, Lange BM, Choi G, Koo YJ, Yoo YJ, Choi YD, Choi G, Browse J. Transcriptional regulators of stamen development in Arabidopsis identified by transcriptional profiling. Plant J. 2006;46(6): 984-1008.

62. Kunieda T, Mitsuda N, Ohme TM, Takeda S, Aida M, Tasaka M, Kondo M, Nishimura M, Hara I. NAC family proteins NARS1/NAC2 and NARS2/NAM in the outer integument regulate embryogenesis in Arabidopsis. Plant Cell. 2008:20(10):2631-42.

63. Liu Y, Sun J, Wu Y. Arabidopsis ATAF1 enhances the tolerance to salt stress and ABA in transgenic rice. J Plant Res. 2016;129(5):955-62.

64. Kou X, Watkins CB, Gan SS. Arabidopsis AtNAP regulates fruit senescence. J Exp Bot. 2012;63(17):6139-47.

65. Yang SD, Seo PJ, Yoon HK, Park CM. The Arabidopsis NAC transcription factor VNI2 integrates abscisic acid signals into leaf senescence via the COR/ RD genes. Plant Cell. 2011;23(6):2155-68. 